\title{
INTEGRAL REPRESENTATION OF LINEAR FUNCTIONALS ON SPACES OF SECTIONS
}

\author{
ANTHONY KAREL SEDA
}

\begin{abstract}
An integral representation is given of functionals on the normed linear space of all sections, with compact support, of a Banach bundle $p: E \rightarrow$ $X$. This representation is shown to be equivalent in a certain sense to the strong lifting property on $X$ and to the disintegration of measures property on spaces $q: Y \rightarrow X$ over $X$.
\end{abstract}

1. Introduction. In the appendix of [5], see Problems B and C there, Gierz and Keimel study the following question. Given a Banach bundle $p: E \rightarrow X$ over a compact Hausdorff space $X$, and a bounded linear functional $\phi$ defined on the Banach space $\Gamma(p)$ of all sections of $p$, find functionals $\eta_{x} \in E_{x}^{*}$ with $\left\|\eta_{x}\right\| \leq 1$ for each $x \in X$ and a measure $\mu$ on $X$ such that $\phi(\sigma)=\int_{X} \eta_{x}(\sigma(x)) d \mu(x)$ for all $\sigma \in \Gamma(p)$. To settle this question, they develop a vector valued form of the RadonNikodym theorem [5, pp. 261-280] under an assumption concerning the existence of strong liftings on $M^{\infty}(X, \mu)$, see $\S 2$. Somewhat similar problems have also been considered by Dinculeanu in the context of spaces of vector fields and Orlicz spaces, see $[2, \S 28-29]$.

The purpose of this note is to obtain the integral representation of $\phi$ above using results developed by us in $[\mathbf{1 0}$ and $\mathbf{1 1}]$. We shall have no need specifically to assume that $X$ is compact, but we will suppose that $X$ is locally compact throughout; moreover, our proof works for real or complex scalars, although we will assume complex scalars for definiteness. For this and the following reasons we believe that our proof has certain merits: (i) It is short. (ii) It quickly reduces the problem to well known results concerning disintegration of measures. (iii) It is conceptual: we embed $\Gamma_{c}(p)$ in a space of continuous functions, extend $\phi$ by the Hahn-Banach theorem and then apply a disintegration theorem. Moreover, we are able to show that the functionals $\eta_{x}$ are $\mu$-almost everywhere uniquely determined whenever $E$ is a separable bundle with continuous norm, see part 3) of Theorem 1. (iv) We show that the "integral representation property" (Theorem 1) is equivalent to some extent to the "disintegration of measures property" which is in turn equivalent to the "strong lifting property", see Remark 1 and Proposition 2. (v) We give an answer to a question posed by Gierz concerning addition of integral representations, see $\S 5$.

These results also have applications to induced representations of locally compact groups in the spirit of [13] and Fell's monographs [3 and 4].

2. Preliminary notions and the statement of the theorem. Let $X$ be a locally compact Hausdorff space. We shall say that $X$ has the strong lifting property

Received by the editors January 8, 1983 and, in revised form, January 24, 1983 and March 24, 1983.

1980 Mathematics Subject Classification. Primary 46G15, 28A50; Secondary 28C05, 55R65.

(C) 1984 American Mathematical Society $0002-9939 / 84 \$ 1.00+\$ .25$ per page 
for all bounded positive Radon measures $\mu \neq 0$ on $X^{1}$, if $M_{R}^{\infty}\left(K, \mu_{K}\right)$ always has a strong lifting $T_{K}$, where $K$ denotes the support of $\mu$ and $\mu_{K}$ its restriction to $K$. We refer to [6 and 7] for details and notation concerning the theory of liftings, and to [1 and 2] for integration theory. The notation and terminology of [6 and 7] is consistent with ours which is that of [1], but the reader should note that the term "integrable" in [2] corresponds to "essentially integrable" in [1]. Likewise, "upper integral" and "negligible" in [2] correspond respectively to "essential upper integral" and "locally negligible" in [1]. For a locally compact space $Z$, we denote by $k(Z)$ the space of all complex valued continuous functions on $Z$ with compact support, and by $C_{0}(Z)$ its uniform norm completion.

Suppose $p: E \rightarrow X$ is a complex Banach bundle over $X$ in the sense of [5]. Denote by $\Gamma_{c}(p)$ the complex vector space of all sections of $p$ which have compact support, endowed with the uniform norm: $\|\sigma\|=\sup _{x \in X}\|\sigma(x)\|$, for $\sigma \in \Gamma_{c}(p)$. Our main result is as follows.

THEOREM 1. Suppose $X$ has the strong lifting property for all bounded positive Radon measures on $X$. Given a bounded linear mapping $\phi: \Gamma_{c}(p) \rightarrow C$, there is a family of linear functionals $\eta_{\phi, x}=\eta_{x}$, with $\eta_{x} \in E_{x}^{*}$ for all $x \in X$, and a bounded positive Radon measure $\mu_{\phi}=\mu$ on $X$ such that

(1) $\left\|\eta_{x}\right\| \leq 1$ for all $x \in X$.

(2) For each $\sigma \in \Gamma_{c}(p)$, the function $\langle\sigma, \eta\rangle$ is $\mu$-integrable and bounded, where $\langle\sigma, \eta\rangle(x)=\eta_{x}(\sigma(x))$ for each $x \in X$, and we have $\phi(\sigma)=\int\langle\sigma, \eta\rangle d \mu$.

(3) If $\xi_{\phi, x}=\xi_{x}$ is another family of functionals satisfying (1) and (2), $E$ has continuous norm and $p: E \rightarrow X$ is separable, see [5], then there is a $\mu$-negligible subset $N$ of $X$ such that $\xi_{x}=\eta_{x}$ for all $x \in X \backslash N$.

REMARK. It is shown in [5] that if $p: E \rightarrow X$ is separable (with $X$ compact), then the $\eta_{x}$ can be chosen so that $\langle\sigma, \eta\rangle$ is Borel measurable for each $\sigma \in \Gamma_{c}(p)$.

The remainder of this section will be denoted to shaping the strong lifting hypothesis into a disintegration of measures form which we wish to apply, see Proposition 1. Specifically, we need to cater for the fact that the support of $\mu$ need not be all of $X$, and this requires some modification of the results of [6 and 7 ].

Suppose $X$ has the strong lifting property described above and let $q: Y \rightarrow X$ be a proper (hence continuous) surjection, where $Y$ is locally compact Hausdorff. We shall refer to $q: Y \rightarrow X$ as a space over $X$. Let $m \neq 0$ be a bounded positive Radon measure on $Y$ and let $\mu=q(m)$. Denote the support, $\operatorname{supp}(\mu)$, of $\mu$ by $K$ and let $K^{\prime}=q^{-1}(K)$. Then by $[2,20.14] m$ is concentrated on $K^{\prime}$; let $m_{K^{\prime}}$ denote the restriction of $m$ to $K^{\prime}$. Since $\mu$ is bounded there is a strong lifting $T_{K}$ of $M_{R}^{\infty}\left(K, \mu_{K}\right)$. Recall, see [7], that a mapping $\lambda: x \rightarrow \lambda_{x}$ of $K$ into the cone $\mathcal{M}_{+}\left(K^{\prime}\right)$ of all positive Radon measures on $K^{\prime}$ is appropriate for $\left(\mu_{K}, T_{K}\right)$ if $T_{K}[\lambda]=\lambda$ and $\langle g, \lambda\rangle$ is essentially $\mu_{K}$-integrable for each $g \in k\left(K^{\prime}\right)$. Here, $\langle g, \lambda\rangle$ denotes the mapping defined on $K$ by $\langle g, \lambda\rangle(x)=\left\langle g, \lambda_{x}\right\rangle=\int g d \lambda_{x}$, and the latter statement concerning $\langle g, \lambda\rangle$ is equivalent to the requirement that $\langle g, \lambda\rangle$ be $\mu_{K}$-measurable and $\mu_{K}(|\langle g, \lambda\rangle|)<+\infty$, where $\mu_{K}$ denotes the essential upper integral determined by $\mu_{K}$, see $[2,11.10]$. Since $\mu$ is bounded, $\mu$ (resp. $\mu_{K}$ ) coincides with the upper

\footnotetext{
${ }^{1}$ By working with the one point compactification of $X$ one can actually assume $X$ compact throughout, provided that it is established that whenever $X$ has this lifting property, then so does $X \cup\{\infty\}$. I am indebted to the referee for observing this, and for suggesting some improvements in notation.
} 
integral $\mu^{*}$ (resp. $\mu_{K}^{*}$ ) [1, Chapter 5, p. 7, Corollary 2]. Hence, $\langle g, \lambda\rangle$ is $\mu_{K}$ integrable, and in fact $\mu_{K}$ (resp. $\mu$ )-locally negligible subsets of $X$ will actually be $\mu_{K}$ (resp. $\mu$ )-negligible. Applying [7, Theorem 3] we obtain a mapping $\lambda: x \rightarrow \lambda_{x}$ appropriate for $\left(\mu_{K}, T_{K}\right)$ and a $\mu_{K}$-locally negligible, and hence negligible, subset $N$ of $K$ such that

(1) $\left\|\lambda_{x}\right\|=1$ for all $x \in K \backslash N$.

(2) $\int g d m_{K^{\prime}}=\int\langle g, \lambda\rangle d \mu_{K}$ for all $g \in k\left(K^{\prime}\right)$.

(3) $\lambda_{x}$ is concentrated on $q^{-1}(x)$ for all $x \in K \backslash N$.

Now $q^{-1}(x)$ is closed and so it follows that $\operatorname{supp}\left(\lambda_{x}\right) \subseteq q^{-1}(x)$ for all $x \in K \backslash N$. Next, we note that $X \backslash K$ is $\mu$-negligible and so, moreover, is $N[\mathbf{1}$, Chapter 5 , p. 82, Corollary 1]. Therefore $M=N \cup X \backslash K$ is $\mu$-negligible. The inclusion map $j: K^{\prime} \rightarrow Y$ is proper and the measure $\nu_{x}=j\left(\lambda_{x}\right) \in \mathcal{M}_{+}(Y)$ restricts to $\lambda_{x}$ on $K^{\prime}$ $[2,21.18]$ and $\operatorname{supp}\left(\nu_{x}\right)=\operatorname{supp}\left(\lambda_{x}\right)[2,20.14]$. In this way $\lambda_{x}$ can be regarded as a measure on $Y$ and $\langle g, \lambda\rangle$ is unchanged and so are (1), (2) and (3) above. Finally, for each $x \in X \backslash K$ put $\lambda_{x}=0_{x}$, the zero measure on $Y$ indexed by $x$. For $g \in k(Y)$ the value of $\langle g, \lambda\rangle(x)$ is zero if $x \in X \backslash K$ and is $\left\langle\left. g\right|_{K^{\prime}}, \lambda\right\rangle(x)$ otherwise. Hence, by $[2,21.11]\langle g, \lambda\rangle$ is $\mu$-integrable and a simple calculation shows that

$$
\int g d m=\int\langle g, \lambda\rangle d \mu \text { for all } g \in k(Y) \text {. }
$$

We may summarize these remarks as follows.

PROPOSITION 1. Suppose $X$ has the strong lifting property for all bounded positive Radon measures on $X$. Then for any space $q: Y \rightarrow X$ over $X$ and bounded positive Radon measure $m$ on $Y$, there is a mapping $\lambda: X \rightarrow \mathcal{M}_{+}(Y)$ and a $\mu$ negligible subset $M$ of $X$, where $\mu=q(m)$, such that

(1) $\left\|\lambda_{x}\right\|=1$ for all $x \in X \backslash M$.

(2) $\langle g, \lambda\rangle$ is $\mu$-integrable and $\int g d m=\int\langle g, \lambda\rangle d \mu$ for all $g \in k(Y)$.

(3) $\operatorname{Supp}\left(\lambda_{x}\right) \subseteq q^{-1}(x)$ for each $x \in X \backslash M$.

REMARK 1. When carefully formulated, the disintegration of measures property as above is actually equivalent to the strong lifting property, see [6 and 7].

3. The proof of Theorem 1. Following [12], let $E^{*}=\bigcup_{x \in X} E_{x}^{*}$, where $E_{x}^{*}$ denotes the dual of $E_{x}$, and let $p^{*}$ denote the canonical projection of $E^{*}$ onto $X$. For each section $\sigma$ of $E$, define $F_{\sigma}: E^{*} \rightarrow C$ by $F_{\sigma}(f)=f\left(\sigma\left(p^{*}(f)\right)\right)$, and endow $E^{*}$ with the weak topology generated by the collection $\Omega$ of all the $F_{\sigma}$ together with $p^{*}$. Let $Y=\bar{S}(E)$ be the subspace of $E^{*}$ consisting of those $f$ with $\|f\| \leq 1$, and let $q$ denote the restriction of $p^{*}$ to $Y$. It was shown in $[12, \S 3]$ that $q: Y \rightarrow X$ is a space over $X$ (and even that $q$ is open) assuming continuity of the norm of $E$. In the case of upper semicontinuous norm we proceed as follows. Firstly, the proof of $[\mathbf{1 2}$, Proposition 4] requires no change since $E$ is full [5, Theorem 3.2]. Therefore $\Omega$ separates points of $E^{*}$ and it follows that $Y$ is Hausdorff. Following the proofs of [12, Propositions 6 and 7], we note that $\|\sigma(\cdot)\|$ is upper semicontinuous, nonnegative and vanishes at $x$; it is therefore continuous at $x$. Thus, it follows that $\hat{G}$ is well defined and linear on $E_{x}$ in our present case. Finally, the argument used to establish $\|\hat{G}\| \leq 1$ needs only minor changes and the use of $[\mathbf{9}$, Corollary 1.2$]$ in place of $[\mathbf{3}$, Proposition 1.5]. Thus, $q: Y \rightarrow X$ is a space over $X$. 
For each $s \in E_{x}$, let $F_{s}$ denote the induced functional on $E_{x}^{*}$ and let $\varepsilon_{x}: s \rightarrow$ $F_{s}$ be the natural embedding of $E_{x}$ in the space $C_{0}\left(Y_{x}\right)=C\left(Y_{x}\right)$ of continuous functions on the fibre $Y_{x}$ of $Y$. Define $T: \Gamma_{c}(p) \rightarrow k(Y)$ by $T(\sigma)=F_{\sigma}$ and note that $\left\|F_{\sigma(x)}\right\|=\|\sigma(x)\|$; it follows that $T$ is a linear isometry.

We note at this point, for convenience, that if the norm of $E$ is continuous, then $q$ is open [12, Proposition 5]. We may therefore construct the Banach bundle $\bar{p}: \bar{A}(Y) \rightarrow X$ as described in $\left[\mathbf{1 1}\right.$ and 12]. Moreover, $k(Y)$ coincides with $\Gamma_{c}(\bar{p})$ under the identification $\theta \rightarrow \tilde{\theta}$, for if $\sigma \in \Gamma_{c}(\bar{p})$, then $\theta$ defined on $Y$ by $\theta(f)=$ $\sigma(q(f))(f)$ is continuous [12, Proposition 3], has compact support and is such that $\tilde{\theta}=\sigma$.

Now suppose $\phi \in \Gamma_{c}(p)^{*}$. By considering $\phi \circ T^{-1}$ we can regard $\phi$ as a functional defined on the subspace $T\left(\Gamma_{c}(p)\right)$ of $k(Y)$. Hence by use of the Hahn-Banach theorem we can now extend $\phi$ to an element $m$ of $k(Y)^{*}$ without changing its norm. Then $m$ is a bounded Radon measure on $Y$. Suppose, initially, that $m$ is in fact positive, and apply Proposition 1 to obtain a mapping $\lambda: x \rightarrow \lambda_{x}$ with the properties listed there. Let $\lambda_{x}^{\prime}$ denote the restriction of $\lambda_{x}$ to $\varepsilon_{x}\left(E_{x}\right)$ and let $\eta_{x}=\lambda_{x}^{\prime} \circ \varepsilon_{x}$ for all $x \in X \backslash M$, otherwise let $\eta_{x}$ be the zero functional in $E_{x}^{*}$. Then $\left\|\eta_{x}\right\| \leq 1$ for all $x$ and conclusion (2) follows. For the general case, we apply $[\mathbf{1}$, Chapter 5, p. 54, Corollary 3] to obtain a universally measurable function $u$ such that $|u|=1$ and $m=u|m|$, where $|m|$ is the least positive measure dominating $m$. Applying the former case to $|m|$ and multiplying $\eta_{x}$ by $u(x)$, we obtain the conclusions (1) and (2) in general up to a slight change in notation.

For the final conclusion, $(3)$, we first note that $\Gamma_{c}(p)$ is a fundamental family of continuous vector fields on $X$ in the sense of $[2, \S 28]$, since in fact some element of $\Gamma_{c}(p)$ passes through any given point of $E$, see [5 or 4, Appendix]. Secondly, [2, 28.7 and 28.2] and [4, Corollary 1, p. 13] show that sections (resp. sections with compact support) of $E$ correspond exactly to vector fields (resp. vector fields with compact support) continuous with respect to $\Gamma_{c}(p)$. Identify the scalar field $C$ with its dual which is then a norming space for $C$. If, now, $\xi_{x}$ and $\eta_{x}$ satisfy (1) and (2), then $x \rightarrow \xi_{x}$ and $x \rightarrow \eta_{x}$ are functional fields which satisfy the hypothesis of $[2,28.27]$, and $\Gamma_{c}(p)$ satisfies Godement's countability axiom. It therefore follows that $\xi_{x}=\eta_{x}, \mu$-almost everywhere, and the proof is complete.

Let $F$ be a Banach space and let $k(X, F)$ denote the space of all continuous functions on $X$ taking values in $F$ and having compact support. Form the product bundle $E=X \times F$ whose projection $p$ is simply the projection on the first factor. Then $k(X, F)$ can be identified with $\Gamma_{c}(p)$. In this case, Theorem 1 yields the following result.

COROLlaRY 1. With the hypothesis on $X$ of Theorem 1, let $\phi$ be a bounded linear functional on $k(X, F)$. Then there is a mapping $\eta: X \rightarrow F^{*}$ and a bounded positive Radon measure $\mu$ on $X$ such that

(1) $\|\eta(x)\| \leq 1$ for all $x \in X$.

(2) For each $f \in k(X, F)$ the function $\langle f, \eta\rangle$ is $\mu$-integrable and bounded, and $\phi(f)=\int\langle f, \eta\rangle d \mu$.

(3) If $F$ is separable and $X$ is second countable, then the function $\eta$ is uniquely determined $\mu$ almost everywhere. 
Proof. Conclusions (1) and (2) follow from Theorem 1. As far as (3) is concerned, if $F$ is separable then it is second countable and so $E=X \times F$ is second countable. Hence by Fell's result $[\mathbf{3}, 1.8] E$ is a separable bundle and (3) also follows from Theorem 1.

It will be convenient to call a space $q: Y \rightarrow X$ over $X$ open if $q$ is an open map. (This coincides with the term "space over $X$ " as used in [12].)

We have already observed that, in a sense, the strong lifting property is equivalent to the disintegration of measures property. Moreover, the proof of Theorem 1 shows that the disintegration of measures property implies the integral representation property of elements $\phi \in \Gamma_{c}(p)^{*}$ for all Banach bundles $p: E \rightarrow X$. Kitchen and Robbins [8, Theorem 2.2] have noted the converse implication in case $X$ and $Y$ are compact metric. We will next establish this converse implication more generally as follows:

PROPOSITION 2. The following two statements are equivalent.

(1) For all open spaces $q: Y \rightarrow X$ over $X$ and all bounded positive Radon measures $m$ on $Y, m$ has a disintegration as in Proposition 1.

(2) For all Banach bundles $p: E \rightarrow X$ with continuous norm, every functional $\phi \in \Gamma_{c}(p)^{*}$ has an integral representation as in Theorem 1 .

ProOF. We have only to establish the implication $(2) \Rightarrow(1)$. So suppose $q: Y \rightarrow$ $X$ is any open space over $X$ and $m$ a bounded positive Radon measure on $Y$. Form the Banach bundle $\bar{p}: \bar{A}(Y) \rightarrow X$ as in $[\mathbf{1 1}]$ and note, again, that $\Gamma_{c}(\bar{p})$ coincides with $\{\tilde{\varphi}, \varphi \in k(Y)\}$. The expression $\phi(\tilde{\varphi})=m(\varphi)$ now determines an element $\phi$ of $\Gamma_{c}(\bar{p})^{*}$. The assumption (2) applied to $\phi$, and thereby to $m$, yields a positive Radon measure $\mu^{\prime}$ on $X$ and positive Radon measures $\lambda_{x}^{\prime}$ on $Y_{x}$ for each $x$ such that $m=\int_{X} \lambda_{x}^{\prime} d \mu^{\prime}(x)$, and $\left\|\lambda_{x}^{\prime}\right\| \leq 1$. Moreover, the $\lambda_{x}^{\prime}$ can be extended to $Y$ as in the proof of Proposition 1 with no other change. Multiplying and dividing by the locally integrable function $x \rightarrow \lambda_{x}^{\prime}(Y)$ we obtain $m=\int_{X} \lambda_{x} d \mu(x)$ with $\left\|\lambda_{x}\right\|=1$ $\mu$-almost everywhere. It follows that $\mu=q(m)$ as required.

4. A generalisation of Theorem 1. A key step in the proof above of Theorem 1 lies in extending $\phi$ defined on the subspace $\Gamma=T\left(\Gamma_{c}(p)\right)$ of $k(Y)$ to $m$ defined on all of $k(Y)$. Suppose now that we endow $\Gamma_{c}(p)$ and $k(Y)$ with the inductive limit topologies of the spaces $\Gamma(p, K)$ resp. $k(Y, K)$ as $K$ runs over the compact subsets of $X$, where $\Gamma(p, K)$ resp. $k(Y, K)$ denotes those elements of $\Gamma_{c}(p)$ resp. $k(Y)$ with support contained in $K$. The requirement then that $\phi$ be continuous on $\Gamma_{c}(p)$ is simply that the restriction of $\phi$ to each $\Gamma(p, K)$ be continuous. Call $\phi$ extendable, if it can be extended to a continuous linear form $m$ on all of $k(Y)$. Conditions for this to hold can be formulated within the theory of topological vector spaces. For example, if we work only with real scalars, then $\Gamma$ is a Riesz space and $\phi$ is relatively bounded. It is, therefore, the difference of two positive linear forms [1, Chapter II, p. 28, Theorem 1], and the problem is reduced to extending positive linear forms. Criteria governing this can be found in $[\mathbf{1 0}$, Chapter 2, §2]. Again, an extension is possible if $\Gamma$ is rich in $k(Y)[2,1.21]$. In any event, whenever this extension is possible $m$ will be, by definition, a Radon measure on $Y$. After suitably modifying Proposition 1 to cope with unbounded measures, we obtain:

THEOREM 2. Suppose $X$ has the strong lifting property for all positive Radon measures on $X$, and suppose $\phi: \Gamma_{c}(p) \rightarrow C$ is a linear form continuous for the 
inductive limit topology. If, further, $\phi$ is extendable, then there exists a family of functionals $\eta_{x} \in E_{x}^{*}(x \in X)$ and a positive Radon measure $\mu$ on $X$ such that

(1) $\left\|\eta_{x}\right\| \leq 1$ for all $x \in X$.

(2) For each $\sigma \in \Gamma_{c}(p)$, the function $\langle\sigma, \eta\rangle$ is essentially $\mu$-integrable and bounded, and $\phi(\sigma)=\int\langle\sigma, \eta\rangle d \mu$.

(3) If $p: E \rightarrow X$ is separable and the norm of $E$ is continuous, then the $\eta_{x}$ are $\mu$-locally almost everywhere uniquely determined.

5. Integral representation and addition of functionals. It will be convenient to write $\phi=\int_{X} \eta_{x} d \mu(x)$ in case $\phi$ has the representation given in Theorem 1 , which we call an integral representation of $\phi$. Gierz [5, p. 3] asks: if

$$
\phi=\int_{X} \eta_{x} d \mu(x) \text { and } \phi^{\prime}=\int_{X} \eta_{x}^{\prime} d \mu^{\prime}(x)
$$

where $\phi, \phi^{\prime} \in \Gamma_{c}(p)^{*}$, how do we add their representations so as to give a representation of $\phi+\phi^{\prime}$ ? We briefly consider this question by proving the following proposition.

PROPOSITION 3. Let $\phi, \phi^{\prime} \in \Gamma_{c}(p)^{*}$. Then there exist integral representations

$$
\phi=\int_{X} \eta_{x} d \mu(x), \quad \phi^{\prime}=\int_{X} \eta_{x}^{\prime} d \mu^{\prime}(x)
$$

of $\phi$ and $\phi^{\prime}$ such that $\phi+\phi^{\prime}$ has an integral representation

$$
\phi+\phi^{\prime}=\int_{X} \eta_{x}^{\prime \prime} d\left(\mu+\mu^{\prime}\right)(x),
$$

for some family $\left\{\eta_{x}^{\prime \prime} ; x \in X\right\}$.

PROOF. Let $m$ and $m^{\prime}$ denote the positive Radon measures on $Y$ determined by the extensions $\phi_{1}$ and $\phi_{1}^{\prime}$ of $\phi$ and $\phi^{\prime}$ resp. as in the proof of Theorem 1. Let $\mu=q(m)$ and $\mu^{\prime}=q\left(m^{\prime}\right)$, then by Theorem 1 we obtain integral representations of $\phi$ and $\phi^{\prime}$ as stated. But $\phi_{1}+\phi_{1}^{\prime}$ is an extension of $\left(\phi+\phi^{\prime}\right)$ and $\mu+\mu^{\prime}=q\left(m+m^{\prime}\right)$ $[2,20.22]$. Hence, the result follows by the proof of Theorem 1 again.

A deeper analysis of this problem along these lines involves questions concerning the uniqueness of $\mu$ and the $\eta_{x}$ in any integral representation. We do not propose to discuss these matters here.

6. Extension of Theorem 1 to $\Gamma_{0}(p)$. Let $p: E \rightarrow X$ be a Banach bundle over $X$ and let $\Gamma_{0}(p)$ denote the space of all sections of $E$ which vanish at infinity. A simple modification of the classical result ${ }^{1}$ that $k(X)$ is dense in $C_{0}(X)$ yields

PROPOSITION 4. The space $\Gamma_{0}(p)$ is the uniform norm completion of $\Gamma_{c}(p)$.

We finish by showing that Theorem 1 can be extended to describe elements of $\Gamma_{0}(p)^{*}$. So suppose $X$ satisfies our usual hypothesis and $\phi \in \Gamma_{0}(p)^{*}$. By restriction $\phi$ determines an element of $\Gamma_{c}(p)^{*}$ and so, by Theorem $1, \phi$ has an integral representation $\phi(\sigma)=\int\langle\sigma, \eta\rangle d \mu$ for all $\sigma \in \Gamma_{c}(p)$. By Urysohn's lemma and the principle of localisation [2, 9.39] it follows that $\langle w, \eta\rangle$ is $\mu$-measurable for all $w \in \Gamma_{0}(p)$. Moreover, $\mu \cdot(|\langle w, \eta\rangle|) \leq\|w\|\|\mu\|$ and so $\langle w, \eta\rangle$ is $\mu$-integrable for each $w \in \Gamma_{0}(p)$. The expression $L(w)=\int\langle w, \eta\rangle d \mu$ now determines a linear form on

\footnotetext{
${ }^{1}$ See for example, W. Rudin, Real and complex analysis, McGraw-Hill, New York, 1966, p. 69.
} 
$\Gamma_{0}(p)$ and $|L(w)| \leq\|w\|\|\mu\|$ so that $L$ is bounded. Also $L$ is an extension to $\Gamma_{0}(p)$ of the restriction of $\phi$ to $\Gamma_{c}(p)$. By Proposition 4 such an extension is unique, and so $\phi(w)=\int\langle w, \eta\rangle d \mu$ for all $w \in \Gamma_{0}(p)$. Hence, we have proved

THEOREM 3. Let $X$ satisfy the strong lifting hypothesis of Theorem 1. Then every element $\phi \in \Gamma_{0}(p)^{*}$ has an integral representation $\phi=\int_{X} \eta_{x} d \mu(x)$.

ACKNowledgement. I am grateful to Professors Gierz, Kitchen and Robbins for supplying me with copies of their work, both published and unpublished.

\section{REFERENCES}

1. N. Bourbaki, Intégration, I-V: Éléments de mathématique, Hermann, Paris, 1965 and 1967.

2. N. Dinculeanu, Integration on locally compact spaces, Noordhoff, Leyden, 1974.

3. J. M. G. Fell, An extension of Mackey's method to Banach*-algebraic bundles, Mem. Amer. Math. Soc. No. 90 (1969).

4. __ Induced representations and Banach*-algebraic bundles, Lecture Notes in Math., Vol. 582, Springer-Verlag, Berlin and New York, 1977.

5. Gerhard Gierz, Bundles of topological vector spaces and their duality, Lecture Notes in Math., Vol. 955, Springer-Verlag, Berlin and New York, 1982.

6. A. and C. Ionescu Tulcea, Topics in the theory of lifting, Ergebnisse der Mathematik und ihrer Grenzgebiete, Vol. 48, Springer-Verlag, Berlin and New York, 1969.

7. __ On the lifting property. III, Bull. Amer. Math. Soc. 70 (1964), 193--197.

8. J. W. Kitchen and D. A. Robbins, Internal functionals and bundle duals, Trinity College and Duke University, Preprint, 1982, pp. 1-14.

9. __ Gelfand representation of Banach modules, Dissertationes Math. 203 (to appear).

10. A. L. Peressini, Ordered topological vector spaces, Harper and Row, New York and London, 1967.

11. A. K. Seda, Banach bundles of continuous functions and an integral representation theorem, Trans. Amer. Math. Soc. 270 (1982), 327-332.

12. $\ldots$, On the categories $\operatorname{Sp}(X)$ and $\operatorname{Ban}(X)$, Cahiers Topologie Géom. Differentielle 24 (1983), 97-112.

13. __ Banach bundles and a theorem of J. M. G. Fell, Proc. Amer. Math. Soc. 83 (1981), 812-816.

Department of Mathematics, University College, Cork, Eire 